venous blood and infarcted myocardium at 5-10 min after retroperfusion. (2) DAPI-positive MSCs were significantly higher in the bFGF+MSCs group than in the MSCs group by immunofluorescence imaging. (3) MSCs were successfully transfected by EGFP and the transfection efficiency was $85 \%$. There were more EGFP-positive endothelial cells $\left(23.8 \pm 6.2 / \mathrm{mm}^{2}\right.$ vs $\left.11.4 \pm 2.9 / \mathrm{mm}^{2}, \mathrm{p}<0.05\right)$ and cardiomyocytes $\left(11.3 \pm 2.5 / \mathrm{mm}^{2} \mathrm{vs}\right.$ $\left.8.3 \pm 2.2 / \mathrm{mm}^{2}, \mathrm{p}<0.05\right)$ in the $\mathrm{bFGF}+\mathrm{MSC}$ group than in the MSCs group by immunofluorescence imaging.

Conclusions Coronary venous retroperfusion was safe. A stable bFGF concentration gradient can be established in vivo between coronary venous blood and infarcted myocardium at 5 to 10 min after retroperfusion, which can promote homing of MSCs into the infarcted myocardium and differentiation.

\section{[gw22-e0667] BASIC FIBROBLAST GROWTH FACTOR PROMOTES IN VIVO HOMING AND DIFFERENTIATION OF MESENCHYMAL STEM CELLS BY CORONARY VENOUS RETROPERFUSION}

Wang Xiao, Nie Shaoping, Qiao Shibin, Zhen Lei, Dong Jianzeng, Liu Xiaohui, Ma ChangshengBeijing Anzhen Hospital, Capital Medical University, Beijing, China

\subsection{6/heartjnl-2011-300867.62}

Objective To elucidate the mechanisms of combined myogenesis and angiogenesis using mesenchymal stem cells (MSCs) and basic fibroblast growth factor (bFGF), we established concentration gradient of bFGF between coronary venous blood and target myocardium by coronary venous retroperfusion along with assessing the effects of bFGF gradient on in vivo homing and differentiation of MSCs.

Methods Acute myocardial infarction (AMI) was induced by ligation of left anterior descending coronary artery. (1) 12 animals were randomly divided into four groups based on the time of balloon dilation (retroperfusion time), being $0 \mathrm{~min}$ $(\mathrm{n}=3), 5 \mathrm{~min}(\mathrm{n}=3), 10 \mathrm{~min}(\mathrm{n}=3), 15 \mathrm{~min}(\mathrm{n}=3)$. One week after AMI, bFGF was retrogradely perfused and bFGF concentrations of serum and myocardial tissue were measured by ELISA. The time of bFGF gradients after coronary venous retroperfusion was evaluated. (2) 12 animals were randomly divided into MSCs group $(n=6)$ and bFGF+MSCs group $(n=6)$ after AMI. MSCs were labelled by 4',6-diamidino-2-phenylindole (DAPI) and one week after implantation, DAPI-positive MSCs in infarcted myocardium were compared between the two groups by immunofluorescence method. (3) 12 animals were randomly divided into MSCs group $(n=6)$ and bFGF+MSCs group $(n=6)$ after AMI. MSCs was cultured and transfected by pLEGFP-N1 vector. 1 week after retroperfusion, EGFP-positive endothelial cells and cardiomyocytes in infarcted myocardium were compared between the two groups by immunofluorescence method.

Results (1) There were statistical differences regarding bFGF concentration between coronary venous blood and infarcted myocardium at $5 \mathrm{~min}(81.13 \pm 15.07 \mathrm{pg} / \mathrm{ml}$ vs $210.66 \pm 65.09 \mathrm{pg} /$ $\mathrm{ml}, \mathrm{p}<0.05)$ and $10 \mathrm{~min}(59.80 \pm 12.15$ vs $124.92 \pm 21.31, \mathrm{p}<0.05)$. Remarkable bFGF gradient can be established between coronary 